\title{
Controller Product Design of Remote Fish Based on Arduino Platform
}

\author{
Jiao Bin ${ }^{*}$
}

School of Art and Design Zhengzhou Institute of Aeronautical Industry Management Zhengzhou, Zhengzhou, Henan Province, 450000, China

\begin{abstract}
A novel controller product design of remote fish based on Arduino Duemilanove platform is proposed. This system is mainly made up of control system and wireless module. The principle diagram of circuit connection between APC-220 and Arduino is investigated. Connection between APC and computer is described in detail. Then the software flow chart of the controller and its corresponding code are also given. The designed product has a certain practical meaning, which can provide reference for the design of controller product.
\end{abstract}

Keywords: Arduino Duemilanove, APC-220 wireless communication module, product design.

\section{INTRODUCTION}

In our real life, there are a lot of remote control products, but most products simulating fishes are driven by propeller, and there is large difference between them and fish's real attitude. An initial and boundary value problem modeling of fishlike swimming was proposed by Jorge San [1]. Experimental performance of a teleoperated robotic fish was proposed by Evangelos Papadopoulos [2]. Nonlinear control methods for planar carangiform robot fish locomotion were proposed by Kristi A [3]. An embedded vision system for robotic fish navigation was proposed by Kai Wang [4].Study on swimming Control of Muliti Mini Robofish was given by Hongxing Wei [5]. Mechatronics design for a robotic fish was proposed by Liu Jindong [6]. Development of multimode biomimetic robotic fish based on central pattern generator was proposed by W. Zhao [7]. This paper designs the controller corresponding to the tail swinging movement. This controller is made up of control system, wireless module, fins and tail. The control module includes power and Arduino Duemilanove. Its function is to control actions of steering gear at tail. Wireless module is composed of APC220 , which is used to receive standard serial data from the host and translate data into electromagnetic wave signal. Fins are used to control directions of fish, so as to achieve the aim of steering. The tail is the main power, and reaction of water makes the fish move. The designed remote fish model is shown in Fig. (1) and Arduino controller is shown in Fig. (2).

Arduino [8] is a piece of simple I/O interface board based on open source code, and has IDE integrated development environment similar to Java and C language, which allows you to quickly use the Arduino language to design interactive work. Arduino can use developed electronic components such as switch, sensors, other controller, LED, stepper motor or other output devices $[9,10]$. Arduino can also become an independent interface to software.

The concrete parameters of Arduino Duemilanove are given as follows.

1. Microcontroller core: AVRmega168-20PU (processing speed can achieve 20 MIPS)

2 . Working voltage: $+5 \mathrm{v}$. It contains all necessary components of the microprocessor, and it can be used simply by inserting a USB cable to computer. ed)

3 . The external input voltage: $+7 \mathrm{v} \sim+12 \mathrm{v}$ (recommend$20 \mathrm{~V}$

4. The external input voltage (extreme); $+6 \mathrm{~V} \leq \mathrm{Vin} \leq+$

5. The digital I/O interface: 14 (including $6 \mathrm{PWM}$ output interfaces)

6. Analog signal input interface: 6

7. DC I/O interface current: $40 \mathrm{~mA}$

8.Flash capacity: $16 \mathrm{~KB}$ (the other $2 \mathrm{k}$ is used for bootloader)

9. An SRAM static storage capacity: $1 \mathrm{~KB}$

10. EEPROM memory capacity: 512 bytes

11. The clock frequency: $16 \mathrm{MHZ}$

Arduino language is established on the basis of $\mathrm{C} / \mathrm{C}++$ language, and in fact it is the basic $\mathrm{C}$ language. Arduino language makes settings of related parameters of AVR microcontroller functional, so that we do not need to understand the bottom layer.

\section{HARDWARE DESIGN OF THE SYSTEM}

The keyboard code is transmitted to CPU of Arduino. After recognizing the action corresponding to keyboard code, Arduino calculates control code of the action and the code is transformed into standard serial data. At last, the control data 


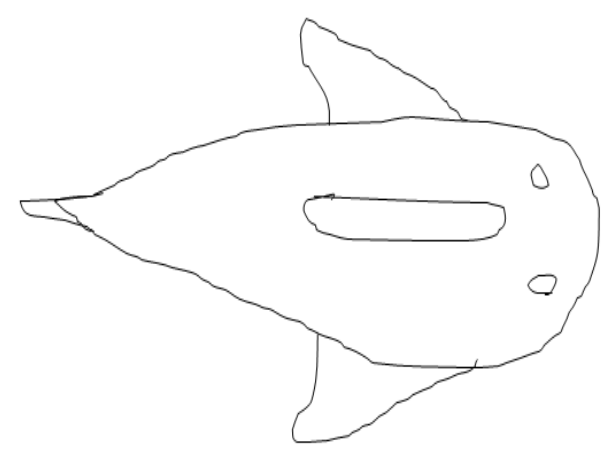

Fig. (1). Designed remote fish model.

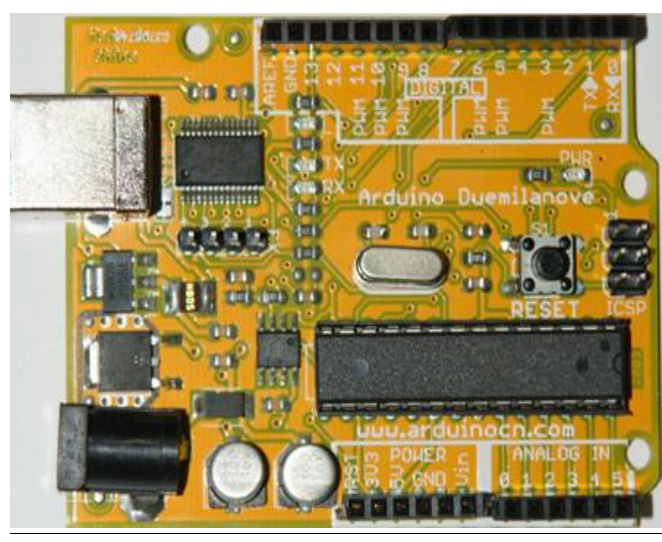

Fig. (2). Arduino controller.

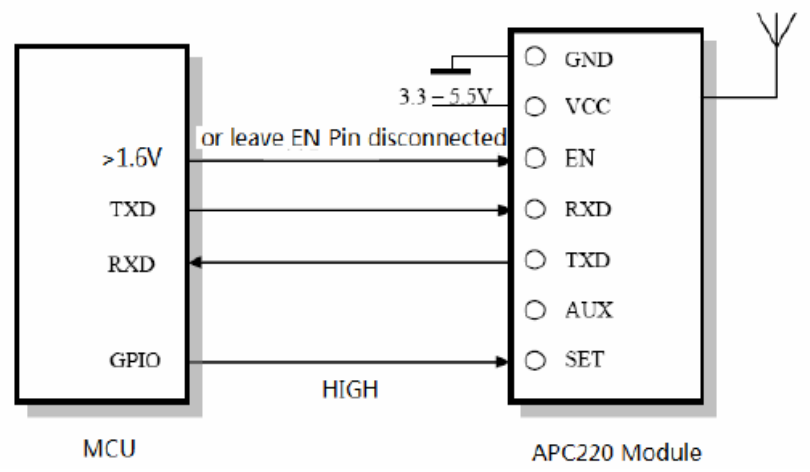

Fig. (3). Connection between MCU and APC220.

is sent out using TX port. There are three steering gears, one is used to provide power and the other two gears are used to change direction.

$\mathrm{RB}-150 \mathrm{MG}$ is a kind of special robot servo steering gear, and the adopted motor is DC brush hollow cup motor. It uses non-iron rotor, which has high energy conversion efficiency, fast response, reliable running, strong adaptive ability and less electromagnetic interference. Compared with the same power motor of iron core, it has small volume and light weight. Feedback potentiometer of steering gear uses the conductive plastic potentiometer, and its accuracy and wear-resisting degree is superior. Integrated circuit for motor control uses digital chip and the power switch H-bridge circuit, and voltage controlled bipolar drive mode has faster response speed, small range of no reaction zone, high positioning accuracy, strong anti-interference ability, good compatibility and other advantages. RB-150MG is better than the traditional steering gear, which is more suitable for the widespread application of robot and airplane model.

Connection between MCU and APC220 is shown in Fig. (3) and connection between APC and computer is shown in Fig. (4). Baud rate setting of APC220 is shown in 


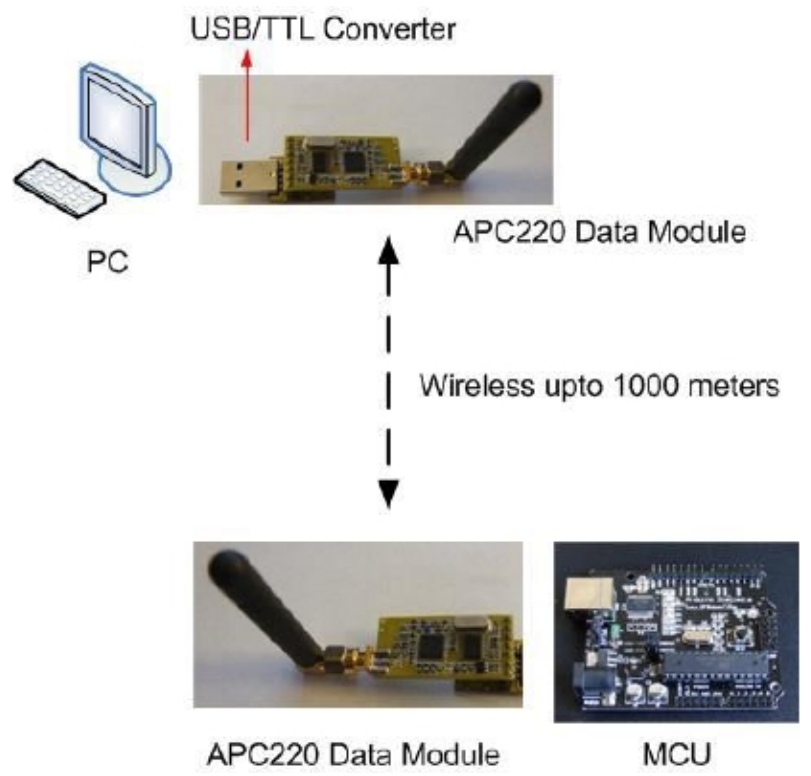

Fig. (4). Connection between APC and computer.

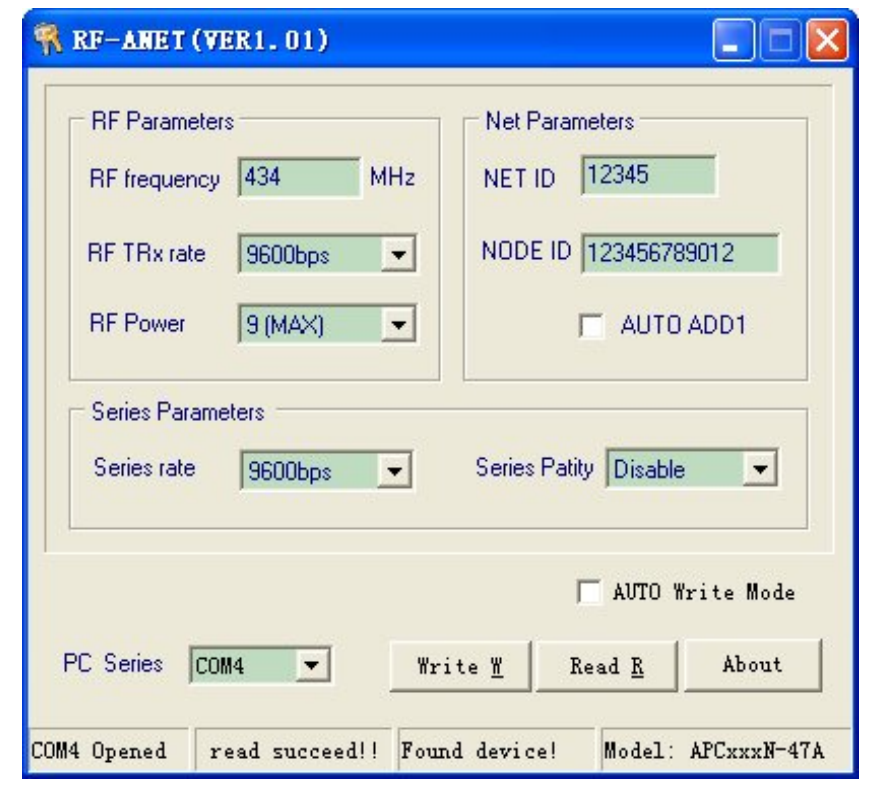

Fig. (5). Baud date setting of APC-220.

Fig. (5) and UART/TTL to RS23S between PC, APC220 module is shown in Fig. (6) and sequence diagram of online settings modification is shown in Fig. (7). APC220 pin and its pin definition are shown in Fig. (8) respectively. After APC is connected with computer, we can use software to set baud rate. Using RF-Magic software to set baud rate is accomplished through UART/TTL port of the module, so it must be connected to computer using UART/TTL to RS232 interface conversion board. We can also use conversion board provided by the company. Firstly, communication lines are connected, open the RF-Magic software, and then open the power supply module. Finally, the module is inserted into the test board. At this time, the status bar of software should show Found Device, and then you can make the appropriate read and write operation. The frequency of APC220 is $434 \mathrm{MHz}$; information transmission rate is $9600 \mathrm{bps}$, transmitting powe is $20 \mathrm{~mW}$, rate of serial port is $1200 \mathrm{bps}$.

The writing setting is : WR 434000 3 9 0 0 (HEX encoding is $0 \times 57,0 \times 52,0 \times 20,0 \times 34,0 \times 33,0 \times 34,0 \times 30,0 \times 30$, 0x30, 0x20, 0x33, 0x20, 0x39, 0x20, 0x30, 0x20, 0x30, $0 \times 0 \mathrm{D}, 0 \times 0 \mathrm{~A})$

\section{Reply: PARA_434000_3_9_0_0}

(HEX encoding is $0 \times 50,0 \times 41,0 \times 52,0 \times 41,0 \times 20,0 \times 34$, 0x33, 0x34, 0x30, 0x30, 0x30, 0x20, 0x33, 0x20, 0x39, 0x20, 0x30, 0x20, 0x30, 0x0D, 0x0A). 
UART/TTL TO RS232

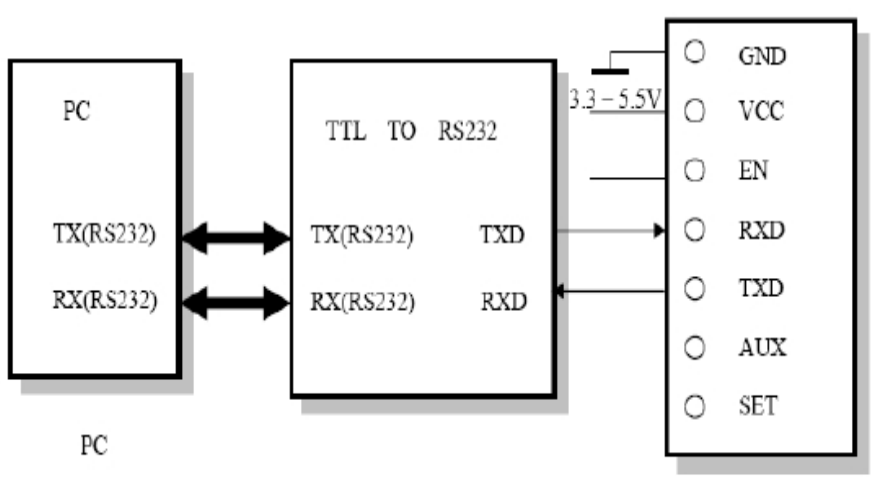

APC220 Module

Fig. (6). UART/TTL to RS232.

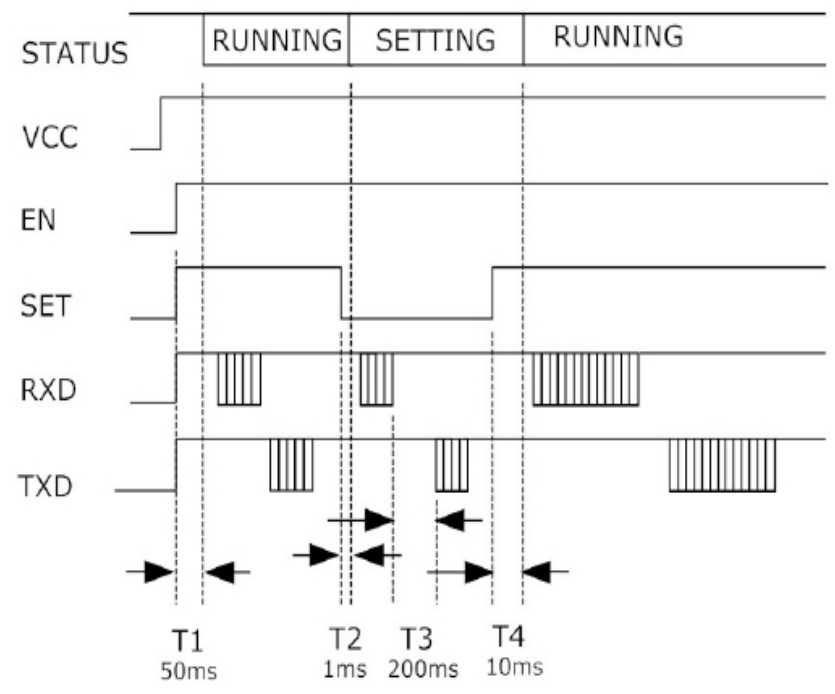

Fig. (7). Sequence diagram of online settings modification.
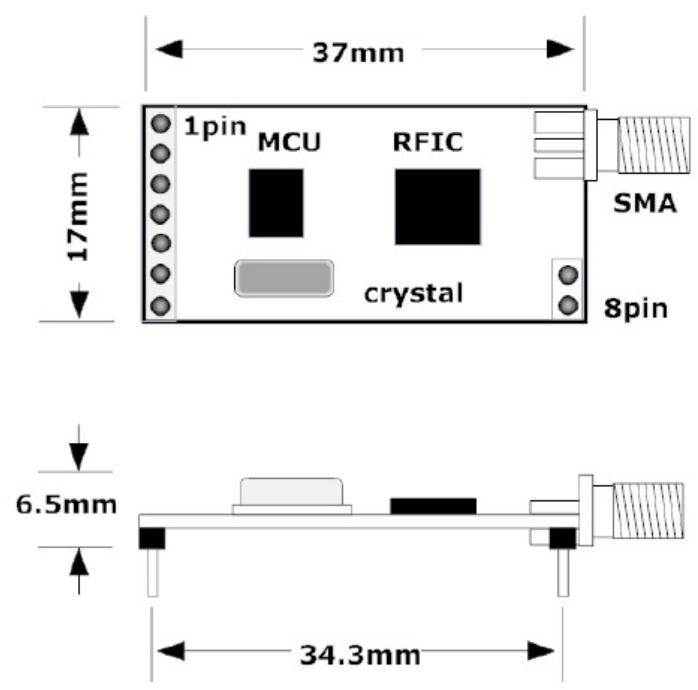

Fig. (8). APC PIN. 


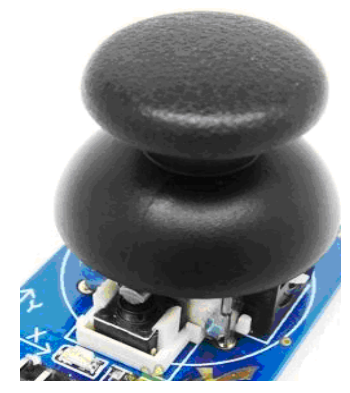

Fig. (9). Joystick module.

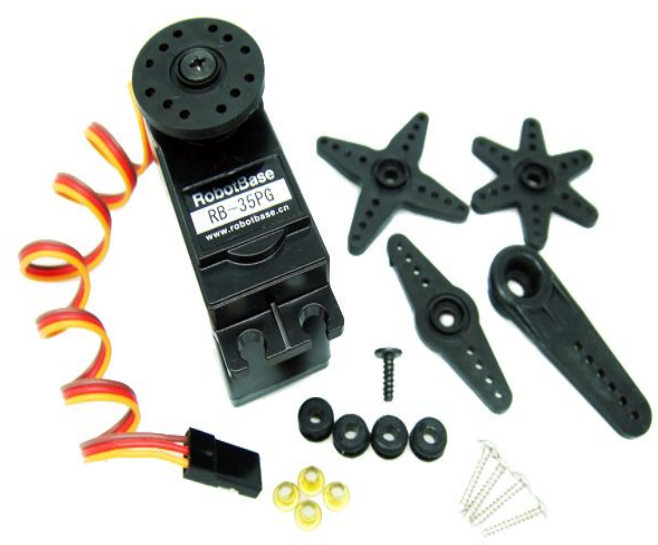

Fig. (10). The steering gear.

Joystick module is shown in Fig. (9). This module includes two analog output interfaces and one digital output interface, and the output value corresponds to $(\mathrm{X}, \mathrm{Y})$ biaxial offsets, the type of which is analog. Button indicates whether the user has pressed on the $\mathrm{Z}$ axis, the type of which is digital. Integrated power light module can display its working state. Accurate positioning identifier can make you easily control motion of objects in two dimension space. Besides, the steering gear is shown in Fig. (10).

\section{SOFTWARE DESIGN}

The used official files are as follows.

Serial.print (75);

Serial.print (75, DEC);

Serial.print (75, HEX);

Serial.print $(75$, OCT);

Serial.print (75, BIN);

Serial.print (75, BYTE);

Serial.println (75);

Serial.println (75, DEC);

Serial.println (75, HEX);

Serial.println $(75$, OCT);

Serial.println (75, BIN);
Serial.println (75, BYTE);

EEPROM block-EEPROM read and write program

Ethernet-Ethernet controller program

LiquidCrystal-LCD control library

Servo-steering gear control library brary

SoftwareSerial-any digital IO port analoging serial li-

Stepper-Stepper motor control library

Wire-library TWI/I2C bus program library

Matrix-LED matrix control library

Sprite-LED matrix image processing control library

The unofficial library files used are described as follows.

DateTime-a library for keeping track of the current date and time in software.

Debounce-for reading noisy digital inputs (e.g. from buttons)

Firmata-for communicating with applications on the computer using a standard serial protocol.

GLCD-graphics routines for LCD based on the KS0108 or equivalent chipset.

LCD-control LCDs (using 8 data lines)

LCD 4 Bit-control LCDs (using 4 data lines) 


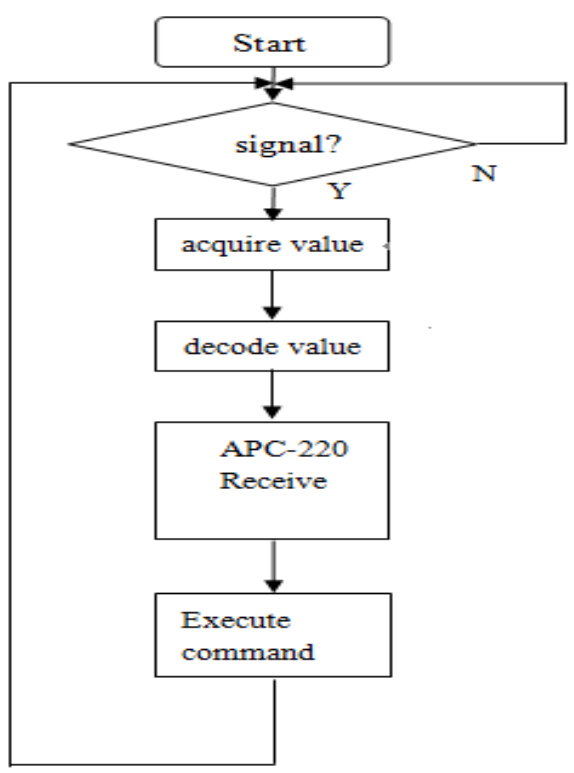

Fig. (11). Flow chart of control code.

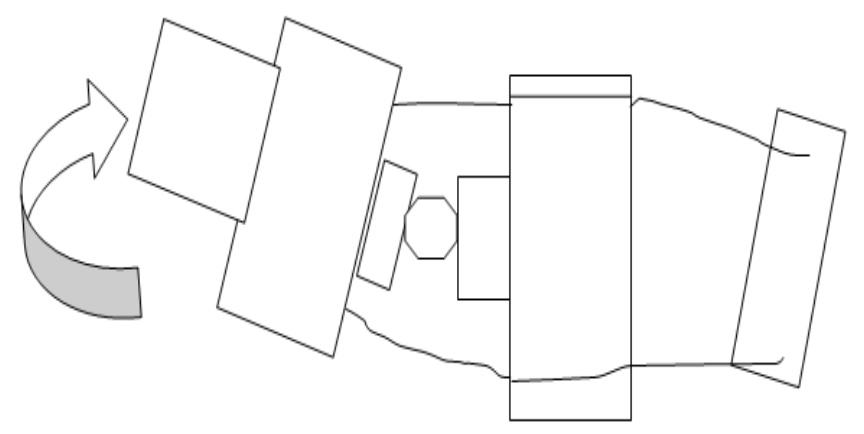

left

Fig. (12). Left sway.

LedControl-for controlling LED matrices or sevensegment displays with a MAX7221 or MAX7219.

LedControl-an alternative to the Matrix library for driving multiple LEDs with Maxim chips.

Messenger-for processing text-based messages from the computer

Metro-help you time actions at regular intervals

MsTimer2-uses the timer 2 interrupt to trigger an action every $\mathrm{N}$ milliseconds.

OneWire-control devices (from Dallas Semiconductor) that use the One Wire protocol.

PS2Keyboard-read characters from a PS2 keyboard.

Servo-provides software support for Servo motors on any pins.
Servotimer1-provides hardware support for Servo motors on pins 9 and 10

Simple Message System-send messages between Arduino and the computer

SSerial2Mobile-send text messages or emails using a cell phone (via AT commands over software serial)

TextString-handle strings

TLC5940-16 channel 12 bit PWM controller.

$\mathrm{X} 10$-Sending X10 signals over AC power lines

Flow chart of control code is shown in Fig. (11). The code can control the fish sways left and right. The schematic diagrams are shown in Fig. (12) and Fig. (13).

The used official files are as follows.

Serial.print(75); 


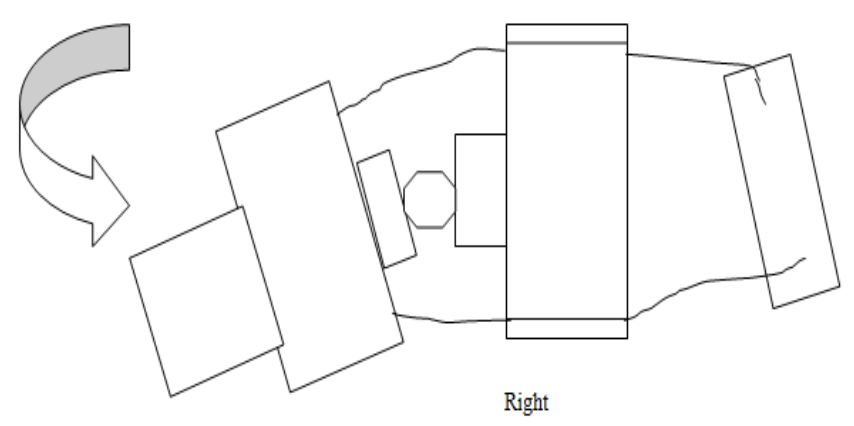

Fig. (13). Right sway.

Serial.print(75, DEC);

Serial.print $(75$, HEX);

Serial.print(75, OCT);

Serial.print $(75, \mathrm{BIN})$;

Serial.print(75, BYTE);

Serial.println(75);

Serial.println(75, DEC);

Serial.println(75, HEX);

Serial.println(75, OCT);

Serial.println(75, BIN);

Serial.println(75, BYTE);

EEPROM block-EEPROM read and write program

Ethernet-Ethernet controller program

LiquidCrystal-LCD control library

Servo-steering gear control library

SoftwareSerial-any digital IO port analoging serial library

Stepper-Stepper motor control library

Wire-library TWI/I2C bus program library

Matrix-LED matrix control library

Sprite-LED matrix image processing control library

The unofficial library files used are described as follows.

DateTime-a library for keeping track of the current date and time in software.

Debounce-for reading noisy digital inputs (e.g. from buttons)

Firmata-for communicating with applications on the computer using a standard serial protocol.

GLCD-graphics routines for LCD based on the KS0108 or equivalent chipset.

LCD-control LCDs (using 8 data lines)

LCD 4 Bit-control LCDs (using 4 data lines)

LedControl-for controlling LED matrices or seven-segment displays with a MAX7221 or MAX7219.

LedControl-an alternative to the Matrix library for driving multiple LEDs with Maxim chips.
Messenger-for processing text-based messages from the computer

Metro-help you time actions at regular intervals

MsTimer2-uses the timer 2 interrupt to trigger an action every $\mathrm{N}$ milliseconds.

OneWire-control devices (from Dallas Semiconductor) that use the One Wire protocol.

PS2Keyboard-read characters from a PS2 keyboard.

Servo-provides software support for Servo motors on any pins.

Servotimer1-provides hardware support for Servo motors on pins 9 and 10

Simple Message System-send messages between Arduino and the computer

SSerial2Mobile-send text messages or emails using a cell phone (via AT commands over software serial)

TextString-handle strings

TLC5940-16 channel 12 bit PWM controller.

X10-Sending X10 signals over AC power lines

Flow chart of control code is shown in figure 11. The code can control the fish sways left and right. The schematic diagrams are shown in figure 12 and figure 13.

The corresponding code is as follows.

\#include $<$ Servo.h $>$

Servo myservo1; // create servo object to control a servo

Servo myservo2; // create servo object to control a servo

Servo myservo3; // create servo object to control a servo

int $\operatorname{pos}=0 ; \quad / /$ variable to store the servo position

int val $=0$;

int $\mathrm{U}=0$;

int $\mathrm{P}=0$;

void setup()

\{

Serial.begin(9600); 
myservo1.attach(9); // attaches the servo on pin 9 to the servo object

myservo2.attach(8); // attaches the servo on pin 9 to the servo object

myservo3.attach(10); // attaches the servo on pin 9 to the servo object

myservo1.write(0);

myservo1.write(90);

myservo2.write $(0)$;

myservo2.write(90);

\}

void $\operatorname{loop}()$

$\{\operatorname{begins}()$;

if(Serial.available ()$>0) / /$ Query whether data appears on the serial port

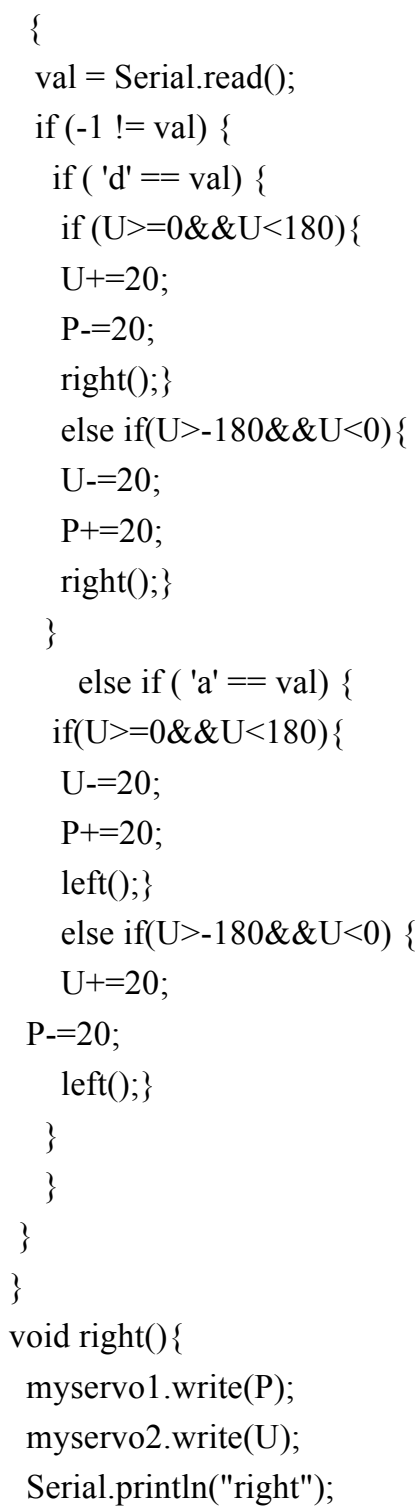

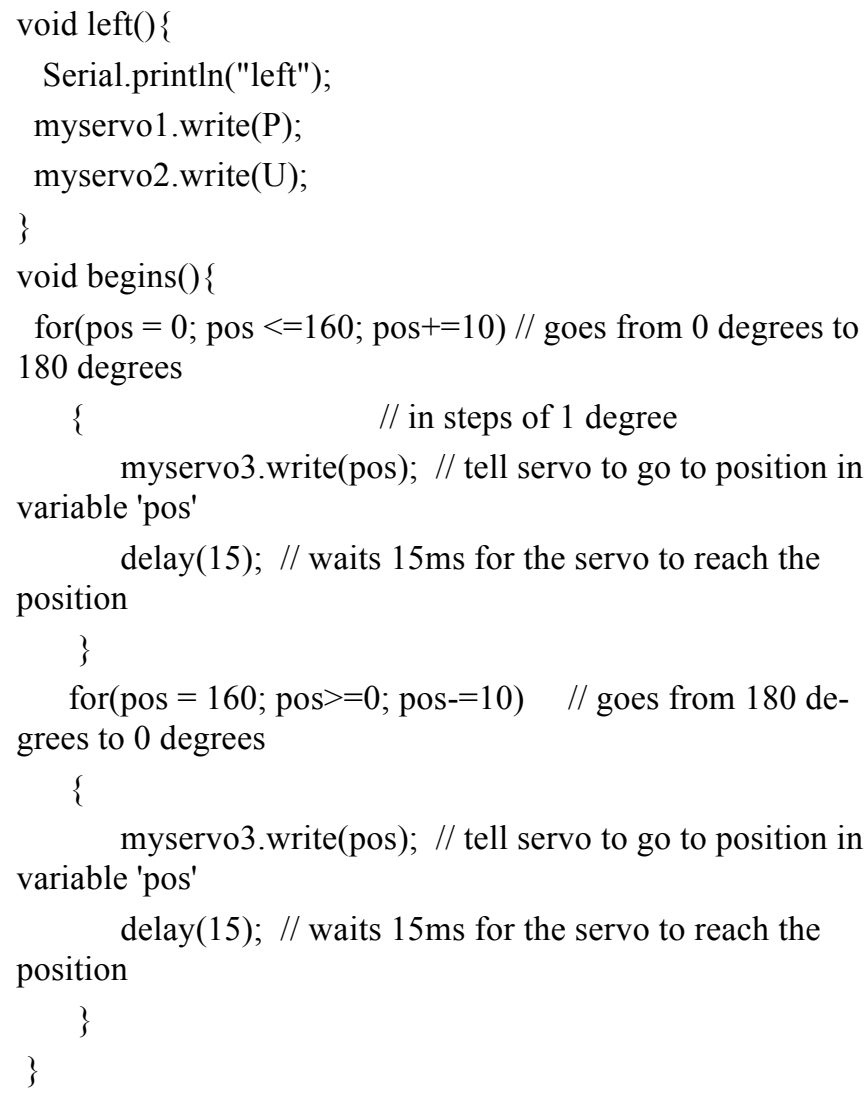

\section{CONCLUSION}

Controller product design of remote fish based on Arduino platform is proposed, First of all, wireless module protocol of APC-220 and connection between APC-220 and Arduino is designed. The specific joystick module is also designed. Then the software flow chart and corresponding code are also given. After the real controller is produced, we see that the designed product has good performance.

\section{CONFLICT OF INTEREST}

The author confirms that this article content has no conflict of interest.

\section{ACKNOWLEDGEMENTS}

This work was supported by the Soft Science Project of the Department of Science and Technology of Henan Province in 2014 (NO. 142400410799).

\section{REFERENCES}

[1] J. S. Martin, J.F. Scheid, T. Takeo, and T. Marius, "An initial and boundary value problem modeling of fishlike swimming," Arch. Rational Mech. Anal, vol. 188, no. 3, pp. 429-455, 2009.

[2] P. Evangelos, A. Efthymios and T. Petros, "Design control and experimental performance of a teleoperated robotic fish," In: Proceedings of $17^{\text {th }}$ Mediterranean Conference on Control \& Automation, Makedonia Palace, Thessaloniki, Greece, 2009, pp. 766-771. 
[3] K.A. Morgansen, D. Vincent, R.. Mason, J. W. Burdick, and R. M. Murray, "Nonlinear control methods for planar carangiform robot fish locomotion," In: Proceedings of IEEE International Conference on Robotics \& Automation, Seoul, Korea, 2002, pp. 427-434.

[4] K. Wang, and J. Yu. "An embedded vision system for robotic fish navigation," In: Proceedings of International Conference on Computer Application and System Modeling, Taiyuan, China, 2010, pp. 333-337.

[5] H. Wei, T. Wang, J. Liang, and M. Liu, "Study on swimming control of muliti mini robofish," In: Proceedings of IEEE International Conference on Information Acquisition, Weihai, Shandong, China, 2006, pp. 207-211.

[6] J. Liu, D. Ian, and H. Hu "Novel mechatronics design for a robotic fish," In: Proceedings of 2005 IEEE/RSJ International Conference on Intelligent Robots and Systems, 2005, pp. 807-812.
[7] W. Zhao, J. Yu, Y. Fang, and L. Wang, "Development of multimode biomimetic robotic fish based on central pattern generator," In: Proceedings of IEEE/RSJ International Conference on Intelligent and Systems, Beijing, China, October, 2006, pp. 3891-3896.

[8] T.B. Huynh, "The testing and design of an arduino microcontroller board for the study of proxemics," University of South Florida St. Petersburg Student Research Journal, vol. 2, no. 1, pp. 1-7, 2010.

[9] W. Durfee, P. Li, and D. Waletzko, "At-home system and controls laboratories," In: Proceedings of 2005 ASEE Annual Conference \& Exposition, Portland, Oregon, 2005

[10] Z. Stanisic, and R. Neimanis, "A new ultra lightweight method for static and dynamic resistance measurements," In: Proceedings of 2010 Conference Record of the IEEE International Symposium on Electrical Insulation (ISEI), San Diego, CA, 2010, pp. 1-4,

(C) Jiao Bin; Licensee Bentham Open.

This is an open access article licensed under the terms of the Creative Commons Attribution Non-Commercial License (http://creativecommons.org/licenses/by-nc/3.0/) which permits unrestricted, non-commercial use, distribution and reproduction in any medium, provided the work is properly cited. 